\title{
Assessment of the usefulness of dihydrotestosterone in the diagnostics of patients with androgenetic alopecia
}

\author{
Izabela Urysiak-Czubatka, Małgorzata L Kmieć, Grażyna Broniarczyk-Dyła \\ Department of General Dermatology, Esthetic and Dermatosurgery, Medical University of Lodz, Poland \\ Head of Department: Prof. Grażyna Broniarczyk-Dyła MD, PhD
}

Postep Derm Alergol 2014; XXXI, 4: 207-215

DOI: 10.5114/pdia.2014.40925

\begin{abstract}
Introduction: Androgenetic alopecia (AGA) is the most common form of hair loss. Clinically observed hair loss is due to the continuous miniaturization of affected hair follicles. Genetic factors and androgenic factors especially dihydrotestosterone (DHT), which is a testosterone tissue metabolite, play major roles in the pathogenesis of AGA. However, expert opinions about the usefulness of DHT in the diagnosis of this type of alopecia are divided.

Aim: To evaluate the usefulness of DHT level in patients with androgenetic alopecia compared with the control group.

Material and methods: The study comprised 49 subjects: 19 women and 9 men with androgenetic alopecia. The control group consisted of 17 healthy women and 4 men without hair loss.

Results: Increased serum concentrations of DHT were observed in patients with androgenetic alopecia (17 women, 5 men), but also in the control group. The differences in mean values of DHT were not significant according to the types of alopecia and the control group. Increased serum concentrations of DHT were not correlated with the advance of alopecia.

Conclusions: Dihydrotestosterone is the most influential androgen and seems to play a very important role in the pathogenesis of androgenetic alopecia. Based on the results of our study and others, the most important factors would appear to be the genetically-determined sensitivity of the follicles to DHT and their different reactions to androgen concentration.
\end{abstract}

Key words: androgenetic alopecia, dihydrotestosterone, diagnostic.

\section{Introduction}

Alopecia (hair loss, shedding) is a dysregulation of the balance between hair loss and regrowth. The hair follicle from which the hair grows is influenced by endogenous and exogenous factors. The hair cycle within the human scalp is asynchronous: adjacent follicles are in various hair cycle periods, and each of them has a microscopic structure characteristic of the current phase. In the development cycle of the hair follicle, there are three phases: active growth (anagen), inhibition of growth and atrophy of the hair follicle (catagen) and resting (telogen). The life cycle of the hair follicle can be compromised in any of its phases. In the human life, the hair follicle passes hair cycle probably on average 20-30 times [1-3]. Hair loss is a physiological process. Daily loss should not exceed 70-100 hairs, but individual variations may exist and increased hair loss is seen in autumn and spring [4]. However, a problem occurs when hair loss exceeds 100 per day and lasts longer than a few weeks. The clinical picture of diseases of the scalp is varied, and the type of hair loss depends on factors that play a role in its pathogenesis.

Alopecia can be divided into non-scarring and scarring forms. Non-scarring alopecia, unlike scarring alopecia, leads to hair loss without destroying hair follicles. The most common types of non-scarring hair loss are male and female androgenetic alopecia, telogen effluvium and alopecia areata. This group includes also some less common variations: hair pulling alopecia, trichotillomania, alopecia syphilitica, and mycosis of the scalp.

Androgens are known to be associated with the mechanisms of hair growth and renewal. The hair follicle is the site of androgen-tissue interaction, and is also

Address for correspondence: Małgorzata L. Kmieć MD, PhD, Department of General Dermatology, Esthetic and Dermatosurgery, Medical University of Lodz, 1 Hallera St, 90-647 Lodz, Poland, phone: +48 4267890 41, +48 696450 708, e-mail: malgorzata.kmiec@umed.lodz.pl Received: 25.06.2013, accepted: 20.08.2013. 
the peripheral organ for the synthesis of substantial amounts of androgens from cholesterol $[5,6]$.

\section{Androgens}

Androgens are hormones synthesized from cholesterol. Under physiological conditions, they are present in both sexes, but their concentration is higher in men. In women, androgens are precursors of female sex hormones and are converted to estrogens.

Androgens can be classified based on the site of production: whether they are synthesized in the testes as testosterone (T), dihydrotestosterone (DHT) or androsterone, in the ovaries as DHT or androstenedione, or in the adrenal cortex as T or dehydroepiandrosterone (DHEA). Androgen receptors are present in almost all organs, which then are influenced by the hormones in both men and women [7]. Secondary sexual features are formed in the uterus, spermatogenesis occurs and muscle mass develops under the influence of androgens. They also have a significant impact on the development and function of the sebaceous glands, and the functioning of the pilosebaceous unit [8]. The response of hair follicles to androgens varies according to body area. More than 200 types of androgenic activity in cells have been reported [9].

The presence of high levels of sex hormones is known as hyperandrogenism. Most often, this is a consequence of excessive production of endogenous hormones by dysregulation of the hypothalamic-pituitary-adrenal cortex, polycystic ovary syndrome (PCOS) or by hormonally-active tumors. Less frequently it arises as a result of exogenous hormone delivery into the body by doping or iatrogenic causes. Hyperandrogenism in women is associated with characteristic features such as formation of a male body type, change in voice tone, the appearance of the male pattern hair, impaired fertility, clitoromegaly, breast atrophy and androgenetic alopecia, while in men, elevated androgen levels usually lead to androgenic alopecia. However, in both sexes, severe seborrhea and acne vulgaris may appear [10].

\section{Dihydrotestosterone}

Dihydrotestosterone is a tissue metabolite of testosterone. It is formed under the influence of the $5 \alpha$-reductase enzyme, which exists as two isoforms. Type $15 \alpha-R$ is mainly found in the sebaceous glands and the epidermis, but is also present in sweat glands, hair follicles, endothelial cells and Schwann cells in the myelin sheaths of nerves [11-14], while type $25 \alpha-R$ is mainly located in the hair follicles (inner layer, infundibulum, sebaceous glands) [15]. The conversion of circulating testosterone into DHT is mainly performed by isoenzyme $5 \alpha$-R type 2 [16]. Dihydrotestosterone has the ability to bind to sex hormone binding globulin (SHBG) more than three times higher than testosterone. In men, approximately $70 \%$ of $\mathrm{DHT}$ is formed from the conversion of testosterone, while in women, the substrate is androstenedione. While elevated concentrations of DHT can be observed in men with androgenetic alopecia or Klinefelter's syndrome, as well as in approximately $40 \%$ of women with idiopathic hirsutism and approximately 35\% with PCOS, decreased concentrations occur in men with azoospermia and anorchia. Determination of DHT concentration is helpful for antiandrogen therapy in patients with prostate cancer or androgenetic alopecia [17].

\section{Androgenetic alopecia}

Androgenetic alopecia concerns both men and women. Male androgenetic alopecia (MAGA) is the most common form of hair loss that occurs in men. It can begin at different stages of life, but its incidence increases with the patient's age. It usually applies to $25 \%$ of men aged 25 years, $40 \%$ of those in their 40 and over $50 \%$ of men aged 50 [16]. While the total incidence of the active form of MAGA in Caucasians over 40 is estimated at 50\%, the symptoms are not as apparent in Asian, Native American and African/Caribbean men $[18,19]$. It is estimated that of all patients experiencing hair loss, androgenetic alopecia is recognized in $95 \%$ of them [3, 20, 21].

Female androgenetic alopecia (FAGA) is one of the most common forms of hair loss in women. Its incidence increases with age; it is estimated that excessive hair loss relates to $12 \%$ of women before 29 years of age, $25 \%$ under 49 and over $50 \%$ before the age of 79 years. Only $43 \%$ of women aged 80 years show no signs of hair loss [22].

The precise etiology and pathogenesis of androgenetic alopecia is still unclear, but genetic and androgenic factors play definite roles.

\section{The genetic factor}

The inheritance of male androgenetic alopecia (MAGA) is not fully understood. However, it is probably an autosomal dominant [23] with variable gene penetrance and multigenic effects dependent on many factors [24, 25]. The probability of MAGA depends on the number of first and second degree relatives who have experienced this type of alopecia. An unfavorable prognostic factor is the presence of androgenetic alopecia (AGA) in the patient's mother or sisters [3]. Garton et al. [26] demonstrated the dependence of MAGA occurrence on the polymorphic gene encoding ornithine decarboxylase, which plays a role in the regulation of the hair cycle. Other studies suggest that genetic variants present in or near the gene encoding the androgen receptor (AR) (chromosome Xq12) may have an impact on the occurrence of MAGA [27-29].

In 2008, Prodi et al. [30] showed that the EDA2R gene (an A2 ectodysplasia receptor), located on chromosome $X$, is associated with MAGA frequency. Although studies suggesting a relationship between MAGA and chromosome $X$ may confirm the impact of genetic factors inher- 
ited from the mother, these results do not explain the observed inheritance of MAGA from the father.

Also in 2008, Hillmer et al. [31] and Brent et al. [32] independently discovered closely related variants of genes on chromosome 20. Men diagnosed with the newly-discovered variant and the other susceptibility locus for the androgen receptor are seven times more likely to experience alopecia. Their presence is dependent on latitude, which explains the ethnic variation of MAGA. Studied variants are located near genes PAX1 and FOXA2. However, their role in hair loss is still not fully understood. Other studies based on genome mapping indicate a relationship between MAGA and chromosome 3q26 [33].

Also, the inheritance of female androgenic alopecia (FAGA) is not fully understood. It may well be of an autosomal dominant nature, with either variable expression of genes or polygenic effects. The wide variation in phenotypic variability, profile of women affected, degree of disease progression and range of hair loss profiles appears to confirm this $[34,35]$. As in men, the pathogenesis of FAGA is based partly on the genes responsible for the production of androgens and their conversion to DHT, as well as the vulnerability of the respective receptors.

The androgen-sensitive miniaturization of the hair follicles is a pathognomonic symptom in both men and women. The presence of two copies of the androgen receptor in women, one of which is randomly inactivated, has raised some controversy about the role of AR in FAGA [36]. So far, only a few genes involved in this form of alopecia have been reported. One of them is the CYP17 gene (10q24.3chromosome) encoding P450 aromatase, which is responsible for, among other things, the release of estradiol and conversion of androgens to estrogens within the hair follicle. The gene was found in numerous studies to be present in patients with PCOS and their brothers, who demonstrated early androgenetic alopecia [19, 36, 37]. Yip et al. [35] also report an increased incidence of the rs4646C allele in women with FAGA: the allele is found within the CYP19A1 gene, which also encodes the aromatase P450. Another gene identified in families with AGA is located on chromosome 3q26 [33].

\section{Influence of androgen}

Two peripherally-circulating androgens which may have an influence on androgenic alopecia are dehydroepiandrosterone sulfate (DHEAS) and androstenedione, which are produced by the adrenal glands. Testosterone and dihydrotestosterone, on the other hand, are synthesized primarily within the gonads [38]. The most important androgen in males is testosterone, formed in the interstitial cells of the testes. However, the major role in the pathogenesis of androgenic alopecia is played by the metabolite dihydrotestosterone, which has a far more potent androgenic action, and is formed by the $5 \alpha$-reductase enzyme ( $5 \alpha-R) .5 \alpha-R$ is present as two isozymes: type $15 \alpha-R$ is encoded by the SRD5A1 gene located on chromosome 5 (p15), while the type 2 SRD5A2 gene is located on chromosome 2 (p23) [39]. In men with genetically determined $5 \alpha$-R2 deficiency, MAGA is absent [40]. Moreover, in men with this form of hair loss, not only is $5 \alpha-R$ more active, but a higher level of DHT can also be found in the frontal compared with the occipital lobe, which is important proof of the participation of this enzyme in the pathogenesis of MAGA [18]. Griffin and Wilson [41], and Sawaya and Price [42] do not support the theory which assumes that elevated levels of androgens play a major role in MAGA, assigning greater significance to the genetically-determined increased sensitivity of hair follicles to the hormones. The level of androgen receptors is 1.5 times higher in the frontal scalp compared to the occipital region. Male pattern alopecia never occurs in the temporal area and the region below the occipital area.

In both men and women with a genetic predisposition, DHT binds to specific androgen receptors. The subsequently-formed complex enters the nucleus of the hair cell and combines with the DNA, thus prompting the activation of genes and production of proteins responsible for the gradual transformation of normal hair follicles to the involutional "miniaturized follicles" $[2,18]$. This transformation takes place by shortening the anagen phase and lengthening the telogen. Gradually, the number of thin telogen hairs increases, while the terminal hairs become thin follicle-type hairs known as vellus hairs [42, 43]. The response of the hair follicles to androgens is varied. The DHT can stimulate the growth of the hair follicles on the face, chest and genital area, while inhibiting the growth of hair in the skin of the scalp. The progression of hair loss varies. It can be very gradual and continuous, but it is often episodic.

In women with a genetic predisposition, androgen-sensitive hair follicles are either over-stimulated or inhibited depending on the part of the body. However, androgen levels in these women may be elevated or normal $[44,45]$. It is estimated that FAGA occurs in only every fourth woman with signs of virilization and elevated levels of androgen [46, 47]. The relationship between the occurrence of FAGA and excessive secretion of 4-androstenedione by the ovaries or the adrenal gland, and increased concentrations of dehydroepiandrosterone (DHEA) and its sulfate (DHEAS), which are synthesized in the adrenal glands, has been demonstrated [4, 45-49]. Androstenedione and DHEA are metabolized circumferentially to testosterone $(T)$, which in turn, is converted to 4 other metabolites, the most important role being played by DHT.

Numerous enzymatic proteins are involved in the enzymatic conversion of androgens, including steroid sulfa-

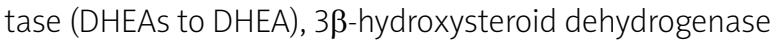
(DHEA to androstenedione) and $17 \beta$-hydroxysteroid oxidoreductase. The latter converts DHEA to androstenediol and the androstenedione to testosterone. In turn, the 
$5 \alpha$-reductase in the cells of the hair follicle is responsible for the conversion of free testosterone to DHT.

While the process of hair loss in men is associated primarily with the excessive activity of $5 \alpha$-R type 2 , both isoenzymes are present in women, but predominantly $5 \alpha-R$ type 1 . An important role is also played by aromatase cytochrome P-450, located in the outer sheath of the hair root, which catalyzes the conversion of androstenedione to estrone and testosterone to estradiol, resulting in lower circulating and tissue levels of $\mathrm{T}$ and DHT. This action explains the clinical differences of androgenetic alopecia in men and women. The concentration of aromatase in women is between 2 and 5 times higher in the skin of the scalp, particularly in the frontal region, which probably determines the retention of hair. In men, lower levels of this enzyme seem to affect hair loss in this region [4, 48-50].

Another hormone which probably takes part in the pathogenesis of FAGA is prolactin. It has been shown that hyperprolactinemia increases DHEAS by its effect on the adrenal cortex [48]. Furthermore, lowered SHBG levels should also be considered as a factor in the pathogenesis of FAGA, insofar that it may be the cause of the increased amount of free testosterone. Some authors believe that a simultaneous reduction in the amount of SHBG and an increase in the $3 \alpha$-glucuronide androstenediol, is a biochemical marker of FAGA. However, other authors consider a combination of decreased SHBG and an increase of T/SHBG ratio to be more significant $[16,51]$.

\section{The clinical picture}

Several classifications have been developed to assess the severity of androgenetic alopecia in men. A scale proposed in 1951 by Hamilton [52] distinguishes 8 degrees of hair loss: I degree indicates completely preserved hairs of the head, II - slight thinning of hair in the leading corners, III - visible thinning of hair in the leading corners (receding hair), IV - deep bend with frontal hair loss and thinning at the top of the head, $V$ - significant hair loss in the frontal area and within the top of the head, VI - partial merging of frontal alopecia lesions and top of the head, and VII and VIII - clear fusion of the two foci of alopecia.

In 1975, this scale was modified by Norwood, who widened its scope, highlighting also the type of angle and peak alopecia (Hamilton-Norwood scale). This scale is the most widely used for clinical assessment of the severity of hair loss in men [53].

In 2007, Lee et al. [54] developed a universal scale to assess the severity of hair loss in men and women: the BASP (basic and specific classification). This classification is based on observation of different types of alopecia. The basic feature (basic-BA) is estimated from the frontal hairline, and the specific type of hair loss (specific-SP) is determined by the density of the hair in the various regions (frontal, top of the head). There are four basic patterns ( $L, M, C$, and $U$ ) and two specific types ( $F$ and $V$ ) of hair loss. The final assessment of the type of hair loss is a compilation of the basic features and characteristic patterns. The clinical course of FAGA can proceed according to either the female or male pattern or sometimes both.

The female alopecia pattern is more common before menopause, and usually relates to women aged 20 to 40 years. Androgen levels are often normal or only slightly elevated [48]. In two thirds of cases, it coincides with polycystic ovary syndrome. This is probably related to the increased production of androgens and their effects on the target tissues $[55,56]$. Hair loss in FAGA women is often widespread. An important clinical symptom is the presence of a parting like "pine branches". Androgen stimulation is responsible for concomitant seborrhea.

The Ludwig classification is most commonly used in assessing the severity of FAGA, and this distinguishes three levels of severity [57]. The first degree is visible thinning in the central part of the scalp while maintaining 2-to 3-centimeter strands of hair on the forehead. In stage II, clear thinning can be seen in the central part of the scalp, and in stage III, alopecia occurs while maintaining the hair in the frontal region. Total hair loss has never been seen.

Another method is the three-point classification developed by Olsen [58], which represents the severity of female androgenetic alopecia, especially in the frontal midline. Androgenetic alopecia in women can also be carried by a male pattern assessed by the 7-point Hamilton-Norwood scale. This pattern of loss is more common in women after the menopause: hair loss is gradual and occurs towards the temporal and parietal area, however, the frontal hair line is preserved $[52,53]$. Other less frequently-used scales are the 5-point Ebling and Sinclair scale, 8-point Savin scale and a universal BASP classification $[51,54,59]$.

\section{Other theories on MAGA pathogenesis}

Among other theories regarding the pathogenesis of androgenetic alopecia, Randall et al. merits particular interest [60]. Their theory assumes that the effect of androgens concerns only one component of the hair follicle - the hair papilla. According to this theory, androgens acting directly on the papilla cells are associated with the androgen receptor, which initiates the expression of the genes for the regulatory factors that in turn, affect other target cells. They take an active role in the paracrine effect and extracellular matrix factors, and influence size changes of the hair papilla and hair follicle.

Ustuner [61] reports that the presence of androgenetic alopecia is a consequence of ischemia and pressure placed on the skin by the hair follicle, which is then pressed against the skull. Over time, in humans, the thickness of the fat decreases and hence, its protective effect on the follicles. In response to the hair loss, the synthesis of testosterone, $5 \alpha-\mathrm{R}$, DHT and DHT receptor 
increases. According to the author, the increase in DHT is not the cause, but the consequence of hair loss, and its action on the hair follicle is limited to transforming the vellus in terminal hair.

\section{Diagnosis of androgenetic alopecia}

In the diagnosis of androgenetic alopecia, data from interview and a characteristic clinical picture are usually sufficient. The most useful criteria in the diagnosis of MAGA include whether hair loss began after puberty, the location of its loss and the presence of thin hair around the top of the head. It is also important to determine its genetic predisposition in closer or more distant relatives. In doubtful cases, a trichogram, consisting of the examination of the roots of the hair, can be performed. Assaying the most important androgen hormones such as testosterone, free testosterone and total dihydrotestosterone is sometimes helpful [62]. Other diagnostic methods include trichoscopy, contrast-enhanced phototrichogram (CE-PTG), a daily assessment of the hair loss, wash test, reflective confocal laser scanning microscopy in vivo (R-CSLM) and trichogram unit area measurement.

The differentiation of male pattern alopecia relates mainly to telogen effluvium, which, however, has a more rapid course and includes areas not typically affected by MAGA. A diagnosis of androgenetic alopecia in women can cause many difficulties. In order to establish the diagnosis, an interview in needed to determine the rate of hair loss and its duration, as well as record any applied treatment and the presence of other dermatological diseases. It is also important to exclude endocrine and gynecological diseases as causes, as well as the intake of any hormone preparations, especially contraceptives. In differentiating the condition from telogen effluvium, it is important to take into consideration any completed surgical interventions or the use of restrictive diets.

In an examination of patients with androgenetic alopecia, the presence of characteristics associated with the coexistence of hyperandrogenism, such as hirsutism, acne, obesity or menstrual disorders, should be noted. If they are present, it is important to record the serum concentrations of free and total testosterone, DHT, DHEA, androstenedione, estradiol and prolactin and SHBG. In order to evaluate the thyroid function, it is also recommended that the concentrations of TSH, FT4 and FT3 are determined. Some authors suggest that further determination of LH, FSH and PSA in serum should then be performed [48, 63-67].

Suitable tests for the diagnosis of FAGA are trichograms, trichoscopies, contrast-enhanced phototrichogram (CE-PTG), and a histopathological examination for the differentiation with CTE. Other less frequently-used methods include an assessment of the daily hair loss, wash test, reflective confocal laser scanning microscopy examination (R-CSLM) or trichogram unit area measurement [68-70].

\section{Aim}

The aim of this study was to evaluate the usefulness of DHT concentrations in patients with androgenetic alopecia (male and female) compared with the control group.

\section{Material and methods}

The study comprised 49 people. The study group consisted of 28 patients experiencing hair loss, receiving treatment from the Department of General Dermatology, Esthetic and Dermatosurgery, Medical University of Lodz, who volunteered during the period from January 2007 to February 2009. The initial diagnosis of alopecia was determined by history and clinical examination. The study group consisted of 19 women and 9 men aged between 17 and 63 years. Children and adolescents under 16 years of age, pregnant women, people with fungal infections of the skin, parasitic infections and immune systemic diseases were excluded from the study.

The control group consisted of 21 healthy volunteers without hair loss, 17 of whom were female and 4 were male. The age of the patients in the control group ranged from 21 to 63 years. A careful history based on the author's own inquiry was taken in all patients, consisting of personal data, age of the patient at the onset of hair loss, the duration and pattern of hair loss (patchy, diffuse), the presence of key factors in determining the cause of hair loss, such as past medical history, stress, lifestyle, improper hair care, medications and family history of alopecia, as well as any details concerning the applied treatment and its efficacy.

During the clinical study, the scalp, hair and skin of the patients were examined, noting signs of seborrhea and the coexistence of hirsutism. The degree of alopecia in men with MAGA was determined based on the 7-point Hamilton-Norwood scale, while in women with FAGA, the determination was based on the 3-point Ludwig classification. In all patients and in the control group, the serum concentrations of dihydrotestosterone were evaluated.

The concentration of DHT in the serum was assessed using a 4132 ElA enzyme immunoassay, reagent kits and DRG International analyzer. The results are given in Table 1.

\section{Statistical analysis}

The results were statistically analyzed using the following tests of significance: the Shapiro-Wilk test, Bartlett's test, one-way ANOVA without replication, the Scheffe multiple comparison test, Mann-Whitney-Wilcoxon test, Fligner-Policello test, logistic regression, $\chi^{2}$ independence test and Fisher's exact test. The confidence interval was set at 95\% (95\% confidence interval - 95\% CI).

The results were considered statistically significant for a $p$-value of less than 5 percent $(p<0.05)$. 
Table 1. Range of standard DHT concentration measurement in serum of the patients ${ }^{\star}$

\begin{tabular}{cc}
\hline Gender & Concentration $[\mathrm{pg} / \mathrm{ml}]$ \\
\hline Female & Premenopausal: $24-368$ \\
\cline { 2 - 2 } & Postmenopausal: $10-181$ \\
\hline Male & 250-990
\end{tabular}

*The standard serum DHT concentrations indicated by the manufacturer were determined only in the United States population.

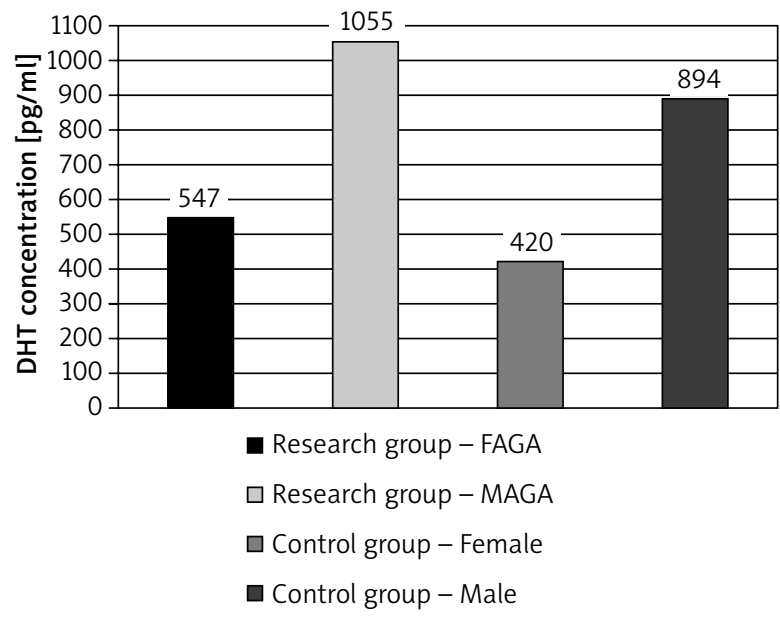

Figure 1. The differences in DHT mean serum in patients with androgenetic alopecia and in the control group

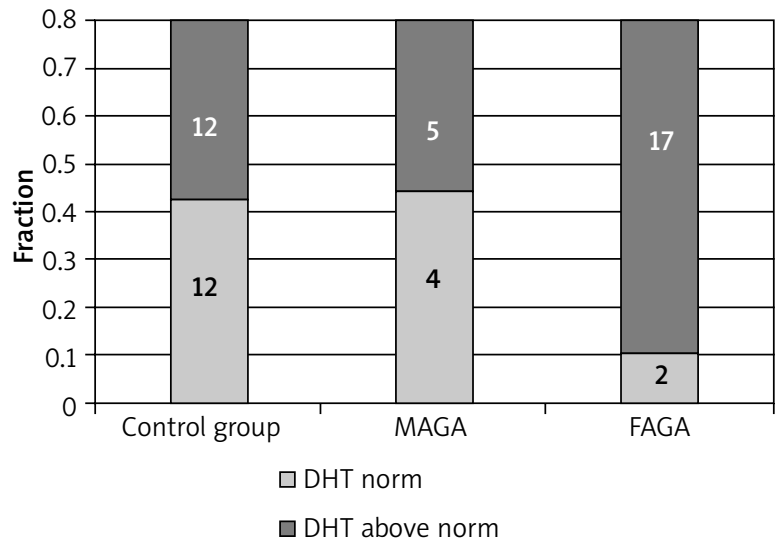

Figure 2. The differences in DHT mean serum in patients with androgenetic alopecia and in the control group

\section{Results}

\section{Results of a clinical trial}

The Ludwig classification was used to assess the severity of hair loss in women with FAGA. On this basis, 5 women were noted as being at stage I, 11 stage II, and 3 stage III alopecia.

Men with MAGA were evaluated with the Hamilton-Norwood classification. Of the nine men examined,
3 were found to be at stage II, 3 other at stage IIIa, and the remaining 3 at stage IV of alopecia.

In the clinical study, clear seborrheic simultaneous changes were found in almost half of the patients in the group with MAGA and FAGA.

\section{The laboratory results}

Serum concentrations of DHT were found to exceed the normal range in the majority of patients and control group subjects. The DHT concentrations were elevated in 17 of 19 women with FAGA, in 5 of the 9 men with MAGA, and in 12 of the 21 patients in the control group ( 1 man, 11 women) (Figures 1, 2).

Differences in mean DHT concentration according to the type of alopecia were not statistically significant relative to the control group. Elevated levels of DHT did not correlate with the progression of hair loss.

\section{Discussion}

Alopecia (hair loss, shedding) is a dysregulation of the balance between hair loss and regrowth. Non-scarring alopecia leads to hair loss without destroying hair follicles. The follicle development cycle may be impaired in any of its phases. The hair follicle is affected by endogenous and exogenous factors. The clinical picture of scalp diseases is varied, the type of hair loss depends on factors that play a role in its pathogenesis.

Despite the fact that every type of alopecia has many characteristic features, their differentiation is sometimes difficult and the results are unclear. So far no reliable diagnostic tools have been developed which would be useful both for evaluating and monitoring the response of therapy in different types of alopecia. Typically, interview data, clinical assessment, laboratory tests, and helper methods such as the pull test, trichogram and trichoscopy, as well as histopathological examination of vertical and horizontal sections, are useful methods in the differentiation of hair diseases. Histopathological examination of the scalp often allows a diagnosis to be made, however, recommendations for its implementation should be carefully considered because of its invasive nature.

Androgenetic alopecia is the most common type of non-scarring hair disorder. Excessive hair loss has been found to have a significantly deleterious effect on the appearance of a patient, often causing distress and social conflict as well as aggravating their quality of life.

In the opinion of some authors, androgens are the most important hormones regulating human hair growth, apart from nutritional deficiency and thyroid disease [71, 72]. There are few studies assessing the correlation between androgenetic alopecia and DHT concentrations in serum samples from the patient. On the basis of studies performed on 52 patients of both sexes with early androgenetic alopecia, Sreekumar et al. [73] found no increase in androgen concentrations (DHT and T), but did note the 
presence of a significantly elevated ratio of DHT/T. However, Vierhapper et al. [74] report that the level of DHT is significantly higher in men with MAGA, and its determination is necessary before anti-androgen treatment. In the present study, the androgen values exceeded the norm, both in patients with androgenetic alopecia, and also in the control group.

Among investigated women with androgenetic alopecia, increased values of DHT were most commonly observed. However, increased levels of DHT did not correlate with the progression of hair loss. The literature reports that although the severity of hair loss may vary, regardless of age, the severity of alopecia is not significant in most cases [34, 35, 41]. Many authors [20, 36, $37,75]$ note the frequent occurrence of this form of hair loss in immediate family members. In the present study, more than half of the patients reported the occurrence of androgenetic alopecia in their mothers, but this was not statistically significant. In some women with FAGA, the coexistence of seborrhea was found, which is also confirmed by other authors [37, 49, 53]. The literature highlights the frequent presence of hirsutism in patients with FAGA, which is also the cause of hyperandrogenism $[10,49,55,63,76]$. However, all patients demonstrated the Ferriman-Gallweya criteria, which did not allow for hirsutism recognition $[63,77]$. The mean age of the group of men with androgenetic alopecia was 25 years. More than half of the respondents with MAGA reported a similar pattern of hair loss in their father or the mother, which may support the theory of a genetic link for this form of hair loss [3, 27-29]. All investigated men reported a history of hormonal disorders. However, 2 patients were taking formulations with carbohydrate-protein containing, among other things, creatine, which causes muscle growth. All patients were either classified as being in stage II, IIIa or IV of hair loss by the Hamilton-Norwood scale. Changes in the nature of acne vulgaris were seen in almost half of the patients.

The results of the present study are consistent those of other authors [56, 78-80], who consider that the level of androgens in women with FAGA may be increased or normal. A retrospective study published in 2009 performed on 228 women with symptoms of hyperandrogenism, also showed higher levels of androstenedione, DHEA and testosterone present in saliva. The SHBG levels were also lower, although their mean values were lower than those in women experiencing hirsutism. An analysis of the results indicated a strong positive correlation of hirsutism with markedly increased hormone levels, particularly testosterone and negative with SHBG. Average total testosterone levels were similar in patients with hirsutism and FAGA [49].

It is noteworthy that biochemical markers of hyperandrogenism can be seen in women with an absence of clinical features. Orme et al. note a complete lack of correlation between FAGA and elevated androgen levels [81]. Schmidt et al. [82] also report no increased androgen levels in patients with FAGA: the level of estrogen was usually within normal limits. A recent Riedel-Baima and Riedel [83] study assessing the concentrations of free and total testosterone, DHEAS, SHBG in 20 women with FAGA also shows a normal range of concentrations of these hormones to be present. Estradiol to free testosterone ratio levels and estradiol to DHEAS ratio levels were found to be significantly lower in FAGA patients compared to the control group. Estradiol to free testosterone ratio levels in women with FAGA were almost always less than 10 . Futterweit et al. and Derksen et al. [55, 84] emphasize that the finding of significantly elevated levels of testosterone and DHEAS may indicate the presence of androgen-secreting tumors or of other diseases which require a precise gynecological and endocrinal diagnosis. According to Price [76], the results of routine hormonal tests performed on women with FAGA are often not much valuable.

According to Stanczyk [85], the determination of the DHT level in serum is not helpful in assessing the prevalence of hyperandrogenism. However, its metabolites, such as sulfate and glucuronide, androstenedione, appear to be clinically relevant markers in the recognition of hirsutism, acne and androgenic alopecia.

Dihydrotestosterone has the most powerful androgen action and seems to play an extremely important role in the pathogenesis of androgenetic alopecia. However, based on the present findings and opinions of many other authors [6, 18, 27-29, 41, 42], the individual, genetically-based sensitivity of hair follicles to DHT concentrations and their varied response to the action of androgens seem to be the most significant factors.

It is worth noting that blood serum DHT concentration is not routinely measured in Polish laboratories: the standard serum DHT concentrations identified by the manufacturers were determined only in the U.S. population.

\section{Conclusions}

Dihydrotestosterone has the strongest androgenic action and seems to play an extremely important role in the pathogenesis of androgenetic alopecia. The usefulness of serum DHT level measurement in the diagnosis of androgenetic alopecia is questionable, especially in women with androgenetic alopecia. In the pathogenesis of androgenetic alopecia, the most important factor seems to be the individual, genetically-based sensitivity of hair follicles to DHT concentration and their varied response to androgen action.

\section{Conflict of interest}

Authors declare no potential conflict of interest. 


\section{References}

1. Kozłowska U, Kozłowska A. Pathophysiology of growth and hair loss [Polish]. Przegl Dermatol 2001; 1: 19-25.

2. Kozłowska U, Kozłowska A. The clinical picture and the treatment of the most common form of alopecia [Polish]. Przegl Dermatol 2001; 4: 311-20.

3. Brzezińska-Wcisło L, Lis-Święty A, Wcisło-Dziadecka D, et al. What's new in the pathogenesis and treatment of androgenetic alopecia in men [Polish]. Derm Klin 2007; 9: 179-81.

4. Randall VA. Androgens and hair growth. Dermatol Ther 2008 21: 314-28.

5. Alsantali A, Shapiro J. Androgens and hair loss. Curr Opin Endocrinol Diabetes Obes 2009; 16: 246-53.

6. Chen W, Thiboutot D, Zouboulis CC. Cutaneous androgen metabolism: basic research and clinical perspectives. J Invest Dermatol 2002; 119: 992-1007.

7. Guay AT. Advances in the management of androgen deficiency in women. Med Asp Hum Sex 2001; 1: 32-8.

8. Szpringer E, Lutnicki K, Zych I. Polycystic ovary syndrome and dysfunction of the pilosebaceous unit in the dermatological practice [Polish]. Wiad Lek 2006; 11-12: 848-54.

9. Balthazart J. Steroid control and sexual differentiation of brain aromatase. J Steroid Biochem Nat Biol 1997; 61: 323-39.

10. Wolf A. Hirsute as interdisciplinary problem - causes, diagnosis and treatment [Polish]. Przegl Dermatol 2008; 6: 523-35.

11. Chen W, Zouboulis CC, Fritsch M, et al. Evidence of heterogeneity and quantitative differences of the type 15 alpha-reductase expression in cultured human skin cells $X$ evidence of its presence in melanocytes. J Invest Dermatol 1998; 110: 84-9.

12. Chen W, Zouboulis CC, Fritsch M, et al. Heterogeneity and quantitative differences of type 15 alpha-reductase expression in cultured skin epithelial cells. Dermatology 1998; 196: $51-2$.

13. Ando $Y$, Yamaguchi $Y$, Hamada K, et al. Expression of mRNA for androgen receptor, 5 alpha-reductase and 17 beta-hydroxysteroid dehydrogenase in human dermal papilla cells. Br J Dermatol 1999; 141: 840-5.

14. Fritsch M, Orfanos CE, Zouboulis CC. Sebocytes are the key regulators of androgen homeostasis in human skin. J Invest Dermatol 2001; 116: 793-800.

15. Bayne EK, Flanagan J, Einstein M, et al. Immunohistochemical localization of types 1 and 25 alpha-reductase in human scalp. Br J Dermatol 1999; 141: 481-91.

16. Tosti A, Camacho-Martinez F, Dawber R. Management of androgenetic alopecia. JEADV 1999; 12: 205-14.

17. Mooradian AD, Morley JE, Korenman SG. Biological actions of androgens. Endocr Rev 1987; 8: 1-28.

18. Hoffman R, Happle R. Current understanding of androgenetic alopecia. Part II: Clinical aspects and treatment. Eur J Dermatol 2000; 10: 407-10.

19. Hoffmann R. Male androgenetic alopecia. Clin Exp Dermatol 2002; 27: 373-82.

20.Shapiro J, Wiseman M, Lui H. Practical management of hair loss. Canad Fam Phys 2000; 46: 1469-77.

21. Wojas-Pelc A, Sułowicz J. Androgenetic alopecia in men and women - pharmacotherapy differences [Polish]. Świat Med Farm 2006; 6: 18-21.

22. Gan DC, Sinclair RD. Prevalence of male and female pattern hair loss in Maryboroug. I Investig Dermatol Symp Proc 2005; 10: $184-9$

23. Carey AH, Waterworth D, Patel K, et al. Polycystic ovaries and premature male pattern baldness are associated with one allele of the steroid metabolism gene CYP17. Hum Mol Genet 1994; 3: 1873-6.

24. Ellis JA, Stebbing M, Harrap SB. Genetic analysis of male pattern baldness and the 5 alpha-reductase genes. J Invest Dermatol 1998; 110: 849.

25. Nyholt DR, Gillespie NA, Heath AC, et al. Genetic basis of male pattern baldness. J Invest Dermatol 2003; 121: 1561-4.

26. Garton RA, McMichael AJ, Sugarman J, et al. Association of a polymorphismin the ornithine decarboxylase gene with male androgenetic alopecia. I Am Acad Dermatol 2005; 52: 535-6.

27. Ellis JA, Stebbing M, Harrap SB. Polymorphism of the androgen receptor gene is associated with male pattern baldness. I Invest Dermatol 2001; 116: 452-5.

28. Hillmer AM, Hanneken S, Ritzmann S, et al. Genetic variation in the human androgen receptor gene is the major determinant of common early-onset androgenetic alopecia. Am Jum Genet 2005; 77: 140-8.

29. Ellis JA, Scurrah KJ, Cobb JE, et al. Baldness and the androgen receptor: the AR polyglycine repeat polymorphism does not confer susceptibility to androgenetic alopecia. Hum Genet 2007; 121: 451-7.

30.Prodi DA, Pirastu N, Maninchedda G, et al. EDA2R is associated with androgenetic alopecia. J Invest Dermatol 2008; 128: 2268-70.

31. Hillmer AM, Brockschmidt FF, Hanneken S, et al. Susceptibility variants for male-pattern baldness on chromosome 20 p11. Nat Genet 2008; 40: 1279-81.

32. Brent JR, Juan X, Geller F, et al. Male-pattern baldness susceptibility locus at 20p11. Nat Genet 2008; 40: 1282-4.

33. Hillmer AM, Flaquer A, Hanneken S, et al. Genome-wide scan and fine mapping linkage study of androgenetic alopecia reveals a locus on chromosome 3q26. Am J Hum Genet 2008; 82: 737-43.

34. Olsen E. Androgenetic alopecia. In: Disorders of hair growth. Olsen E (ed.)., McGraw-Hill, New York 1994; 257-84.

35. Yip L, Zaloumis S, Irwin D, et al. Gene-wide association study between the aromatase gene (CYP19A1) and female pattern hair loss. Br I Dermatol 2009; 161: 289-94.

36. Lyon MF. The William Allan memorial award address: X-chromosome inactivation and the location and expression of X-linked genes. Am J Hum Genet 1988; 42: 8-16.

37. Sinclair RD, Dawber RP. Androgenetic alopecia in men and women. Clin Dermatol 2001; 19: 167-78.

38.Zouboulis CC, Degitz K. Androgen action on human skin from basic research to clinical significance. Exp Dermatol 2004; 13 Suppl 4: 5-10.

39. Russell DW, Wilson JD. Steroid 5alpha-reductase: two genes/ two enzymes. Annu Rev Biochem 1994; 63: 25-61.

40.Imperato-McGinley J, Peterson RE, Gautier T, et al. Hormonal evaluation of a large kindred with complete androgen insensitivity: evidence for secondary 5 alpha reductase deficiency. J Clin Endocrinol Metabol 1982; 54: 931.

41. Griffin JE, Wilson JD. The resistance syndromes: 5 alpha reductase deficiency, testicular feminisation and related disorders. In: The metabolic basis of inherited disease. McGraw-Hill, New York 1989; 1919-44.

42. Sawaya ME, Price VH. Different levels of 5alpha-reductase type I and II, aromatase, and androgen receptor in hair follicles of woman and men with androgenetic alopecia. J Invest Dermatol 1997; 109: 296.

43. Guarrera M, Rebora A. Anagen hairs may fail to replace telogen hairs in early androgenetic female alopecia. Dermatol 1996; 192: 28-31. 
44.Braun-Falco O, Plewig G, Wolff HH, et al. Hair diseases [Polish]. In: Dermatology. Vol. 2. Gliński W, Wolska H (eds.). Czelej, Lublin 2004; 1035-72.

45. Restrepo R, Mckee PH, Calonje E. Diseases of the hair. In: Pathology of the skin with clinical correlations. Vol. 2. McKee PH (ed.). Elsevier Mosby, Philadelphia 2005; 1069-71.

46.Brzezińska-Wcisło L, Szeremeta-Bazylewicz G. Diffuse hair loss in women classification and views on the etiopathogenesis [Polish]. Wiad Lek 1999; 52: 386-92.

47. Kaszuba A, Sulik M, Halbina A. Androgenetic alopecia ethiology, clinical picture and selected treatment methods [Polish]. Ord Lek 2002; 2: 33-7.

48. Camacho-Martinez FM. Hair loss in women. Semin Cutan Med Surg 2009; 28: 19-32.

49. Karrer-Voegeli S, Rey F, Reymond MJ, et al. Androgen dependence of hirsutism, acne, and alopecia in women: retrospective analysis of 228 patients investigated for hyperandrogenism. Medicine 2009; 88: 32-45.

50. Venning VA, Dawber RP. Patterned androgenic alopecia in women. J Am Acad Dermatol 1988; 18: 1073-7.

51. Ludwig E, Montagna W, Camacho F. Female androgenetic alopecia. In: Trichology: diseases of pilosebaceous follicle. Camacho F, Montagna W (eds.). Aula Medica Group, Madrid 1997; 343-55.

52. Hamilton JB. Patterned loss of hair in man; types and incidence. Ann N Y Acad Sci 1951; 53: 708-28.

53. Norwood OT. Male pattern baldness: classification and incidence. South Med J 1975; 68: 1359-65.

54. Lee WS, Ro BI, Hong SP, et al. A new classification of pattern hair loss that is universal for men and women: basic and specific (BASP) classification. J Am Acad Dermatol 2007; 57: 37-46.

55. Futterweit W, Dunaif A, Yeh HC, et al. The prevalence of hyperandrogenism in 109 consecutive female patients with diffuse alopecia. J Am Acad Dermatol 1988; 19: 831-6.

56. Cela E, Robertson C, Rush K, et al. Prevalence of polycystic ovaries in women with androgenic alopecia. Eur J Endocrinol 2003; 149: 439-42.

57. Ludwig E. Classification of the types of androgenetic alopecia (common baldness) occurring in the female sex. Br J Dermatol 1977; 97: 247-54.

58. Olsen EA. The midline part: an important physical clue to the clinical diagnosis of androgenetic alopecia in women. J Am Acad Dermatol 1999; 40: 106-9.

59. Yip L, Sinclair R. Antiandrogen therapy for androgenetic alopecia. Expert Rev Dermatol 2006; 1: 261-9.

60. Randall VA, Thornton MJ, Hamada K, et al. Androgens and the hair follicle. Cultured human dermal papilla cells as a model system. Ann N Y Acad Sci 1991; 26: 355-75.

61. Ustuner ET. Baldness may be caused by the weight of the scalp: gravity as a proposed mechanism for hair loss. Med Hypotheses 2008; 71: 505-14.

62. Mulinari-Brenner F, Bergfeld WF. Hair loss: an overview. Dermatol Nurs 2001; 13: 269-72.

63. Szpringer E. Dermatological consequences of hyperandro genism in women with PCOS. Treatment of disorders of the pilosebaceous unit and hirsutism [Polish]. Derm Estet 2009; 11: 79-90.

64.Deloche C, Bastien P, Chadoutaud S, et al. Low iron stores: a risk factor for excessive hair loss in non-menopausal women. Eur J Dermatol 2007; 17: 507-12.

65. Melegos DN, Yu H, Ashok M, et al. Prostate-specific antigen in female serum, a potential new marker of androgen excess. J Clin Endocrinol Metab 1997; 82: 777-80.
66. Negri C, Tosi F, Dorizzi R, et al. Antiandrogen drugs lower serum prostate-specific antigen (PSA) levels in hirsute subjects: evidence that serum PSA is a marker of androgen action in women. J Clin Endocrinol Metab 2000; 85: 81-4.

67. Thiedke CC. Alopecia in women. Am Fam Physician 2003; 1: 1007-14.

68. Olszewska M, Rudnicka L, Rakowska A, et al. Advances in the diagnosis of alopecia [Polish]. Przegl Dermatol 2009; 96: 247-53.

69. Olszewska M, Rudnicka L. Videodermoscopy as tool for differential diagnosis and monitoring of hair loss. Exp Dermatol 2006; 15: 5.

70. Rudnicka L, Olszewska M, Rakowska A. In vivo reflectance confocal microscopy: usefulness for diagnosing hair diseases. J Dermatol Case Rep 2008; 2: 55-9.

71. Rushton DH, Ramsay ID, James KC, et al. Biochemical and trichological characterization of diffuse alopecia in women. Br J Dermatol 1990; 123: 187-97.

72. Rushton DH. Nutritional factors and hair loss. Clin Exp Dermatol 2002; 27: 396-404.

73. Sreekumar G, Pardinas J, Wong CQ, et al. Serum androgens and genetic linkage analysis in early onset androgenetic alopecia. J Invest Dermatol 1999; 113: 277-9.

74. Vierhapper H, Nowotny P, Maier H, et al. Production rates of dihydrotestosterone in healthy men and women and in men with male pattern baldness: determination by stable isotope/dilutionand mass spektrometry. J Clin Endocrinol Metab 2001; 86: 5762-4.

75. Kuster W, Happle R. The inheritance of common baldness: two B or not Two B? J Am Acad Dermatol 1984; 5: 921-6.

76. Price VH. Androgenetic alopecia in women. J Investig Dermatol Symp Proc 2003; 8: 24-7.

77. Blume-Petyawi U, Hillman K, Guarrera M. Hair growth assessment techniques. In: Hair growth and disorders. Springer-Verlag, Berlin 2008; 125-57.

78. Shum KW, Cullen DR, Messenger AG. Hair loss in women with hyperandrogenism: four cases responding to finasteride. J Am Acad Dermatol 2002; 47: 733-9.

79. Thai KE, Sinclair RD. Finasteride for female androgenetic alopecia. Br J Dermatol 2002; 147: 812-3.

80. Trueb RM. Finasteride treatment of patterned hair loss in normoandrogenic postmenopausal women. Dermatology 2004; 209: 202-7.

81. Orme S, Cullen DR, Messenger AG. Diffuse female hair loss: are androgens necessary? Br J Dermatol 1999; 141: 521-3.

82. Schmidt JB, Lindmaier A, Trenz A, et al. Hormone studies in females with androgenic hair loss. Gynecol Obstet Invest 1991; 31: 235-9.

83. Riedel-Baima B, Riedel A. Female pattern hair loss may be triggered by low oestrogen to androgen ratio. Endocr Regul 2008; 42: 13-6.

84.Derksen J, Nagesser SK, Meinders AE, et al. Identification of virilizing adrenal tumors in hirsute women. N Engl J Med 1994; 331: 968-73.

85. Stanczyk FZ. Diagnosis of hyperandrogenism: biochemical criteria. Best Pract Res Clin Endocrinol Metab 2006; 20: 177-91. 